\title{
Mucosal Incision-Assisted Endoscopic Biopsy as an Alternative to Endoscopic Ultrasound-Guided Fine-Needle Aspiration/Biopsy for Gastric Subepithelial Tumor
}

\author{
Cheol Woong Choi ${ }^{1}$ and Joo Ha Hwang ${ }^{2}$ \\ ${ }^{1}$ Department of Internal Medicine, Pusan National University School of Medicine and Research Institute for Convergence of Biomedical \\ Science and Technology, Pusan National University Yangsan Hospital, Yangsan, Korea, ${ }^{2}$ Division of Gastroenterology and Hepatology, \\ Department of Medicine, Stanford University Medical Center, Stanford, CA, USA
}

See "Reasons for Diagnostic Failure in Forty-Five Consecutive Mucosal Cutting Biopsy Examinations of Gastric Subepithelial Tumors" by Yoshiko Nakano, Toshitatsu Takao, Yoshinori Morita, et al., on page 575-582.

Gastric subepithelial tumor (SET) develops beneath the mucosa or at the extraluminal compression by an intra-abdominal organ. ${ }^{1}$ However, majority of gastric SETs are asymptomatic, measuring $<10 \mathrm{~mm}$ at the time of diagnosis. Therefore, deciding which gastric SETs require additional management remains difficult. Its basic assessment should be performed during the initial endoscopic examination where the SET is first identified. Several endoscopic findings such as the presence of a pillow/cushion sign, certain surface colors (yellowish for lipoma, transparent for cystic lesions, or bluish for vessel structures), or umbilication (suggestive of an ectopic pancreas) are biomarkers indicating a benign lesion. For gastric SETs with these benign endoscopic features, resection is generally unnecessary. ${ }^{1}$ However, determining whether a SET is truly benign or has malignant potential such as gastrointestinal stromal tumor, lymphoma, neuroendocrine tumor, metastatic carcinoma, or adenocarcinoma is often difficult; thus, a definitive

Received: July 11, 2020 Revised: August 11, 2020

Accepted: August 11, 2020

Correspondence: Joo Ha Hwang

Division of Gastroenterology and Hepatology, Department of Medicine, Stanford University Medical Center, 300 Pasteur Dr. H0268, MC:5244, Stanford, CA 94305, USA

Tel: +1-650-497-6313, Fax: +1-650-498-6323, E-mail: jooha@stanford.edu ORCID: https://orcid.org/0000-0002-7534-230X

(c) This is an Open Access article distributed under the terms of the Creative Commons Attribution Non-Commercial License (http://creativecommons.org/ licenses/by-nc/3.0) which permits unrestricted non-commercial use, distribution, and reproduction in any medium, provided the original work is properly cited. diagnosis is needed to determine the next management steps. Known high-risk endoscopic characteristics of malignancy include a tumor diameter of $\geq 20 \mathrm{~mm}$, surface mucosal changes (ulceration or irregular surface), and interval growth between endoscopic examinations. ${ }^{1}$

Although an endoscopic ultrasound (EUS) provides important information such as the differentiation between intramural and extramural lesions, tumor size, layer of origin, and echogenicity, ${ }^{1}$ its interpretation can be operator dependent. As EUS findings of benign gastric SETs such as leiomyoma, schwannoma, and ectopic pancreas may be similar with that of potentially malignant SETs, such as hypoechoic echotexture and location in the submucosa or muscularis propria layer, ${ }^{1}$ the diagnostic accuracy of EUS without tissue acquisition has been unsatisfactory $(48 \%-63.3 \%))^{2,3}$ Therefore, a tissue sample should be obtained to diagnose gastric SETs in order to determine the appropriate management.

Currently, EUS-guided fine needle aspiration and biopsy (EUS-FNA/B) has been regarded as the gold standard for the tissue acquisition of gastric SETs. The overall diagnostic rate of EUS-FNA/B for gastric SETs has been reported as $74.3 \%-83.0 \%$ with low procedure-related complication rates. ${ }^{4}$ The diagnostic adequacy of EUS-FNA/B depends on several factors, including the tumor size, tumor location, endoscopist's expertise/technique, needle type, and availability of an on-site cytologist. ${ }^{4}$ In addition, EUS-FNA/B is not always accessible, especially in resource-limited settings. For these reasons, vari- 
ous alternative endoscopic techniques using more familiar endoscopic techniques have been tried to use in obtaining tissues from gastric SETs. Simple endoscopic forceps biopsy of the normal overlying mucosa is usually not a diagnostic method for gastric SETs because the main tumor develops beneath it.

In this issue of Clinical Endoscopy, Nakano et al. ${ }^{5}$ reported results of 45 consecutive mucosal cutting biopsy examinations for gastric SETs. After making a 10-15-mm mucosal incision using a needle-knife, tissue samples were collected using the endoscopic forceps biopsy. A definitive or suspected diagnosis was possible in 35 of 45 patients (78\%). Several similar mucosal incision-assisted biopsy techniques for gastric SETs such as the unroofing technique or single-incision needle-knife biopsy have been previously reported. ${ }^{6-9}$ A prospective comparative study of EUS-FNA/B and unroofing biopsy in Korea reported no significant difference in the diagnostic yield between the two methods. ${ }^{10}$ EUS-FNA/B showed a shorter procedure time and more successful diagnosis for the larger SETs $(\geq 20 \mathrm{~mm})$ as compared with unroofing biopsy. ${ }^{10}$ Although majority of previous studies were small case series, the overall diagnostic yield of the mucosal incision-assisted biopsy for SETs was reported as $89 \%{ }^{6}$ The diagnostic yield for smaller SETs $(<20$ $\mathrm{mm}$ ) was higher than EUS-FNA/B. ${ }^{9}$ In their analysis, Nakano et al. ${ }^{5}$ determined that the main reason for the failure to obtain adequate tissues for the diagnosis is the failure to expose the tumor. The important advantage of this technique is that the tumor beneath the mucosa can be directly observed during the biopsy using endoscopic forceps. Therefore, before performing mucosal incision-assisted biopsy for SETs, several predictable factors should be considered. First, all therapeutic endoscopists should possess the ability to manage procedure-related bleeding and perforation that might occur during the endoscopic procedure. Failure to control procedure-related adverse events will result in poor endoscopic visualization of deep tumors to obtain proper biopsies. Nakano et al. ${ }^{5}$ reported that bleeding during or after the procedure could be easily controlled by standard endoscopic hemostasis maneuvers. Although this study reported one case of perforation at the greater curvature of the fornix during the biopsy, it can be avoided by careful mucosal incision and biopsy from the top of the gastric SET, not from the base of the lesion. The tumor location in the stomach results in the technical difficulty of the procedure. When SETs are located in the fundus or upper third of the stomach, maintaining the endoscope at the proper position using endoscopic surgical knives can be difficult and blood often pools in this area limiting the tumor exposure after a mucosal incision. For these tumors, a multi-bending endoscope with two independent curving segments can be beneficial, and a water-jet endoscope with a therapeutic channel can help con- trol bleeding more rapidly. During the endoscopic resection, a submucosal fluid cushion should be established to prevent gastric wall perforation. However, submucosal fluid injection before the mucosal incision may result in gastric SET flattening or sinking around the submucosal cushion and thus may become an obstacle for tumor exposure beneath the mucosa. Finally, patient cooperation during an endoscopic procedure under conscious sedation is important for successful endoscopic procedures. If a procedure is expected to be longer than usual or a patient has a paradoxical response after the sedative injection prior the endoscopic examination, monitored anesthesia care or general anesthesia should be considered.

Although EUS-FNA/B has been accepted as the current standard for obtaining tissue samples to diagnose gastric SETs, the variability of diagnostic accuracy and higher level of expertise are required to perform EUS-FNA/B as the conventional endoscopy has been an obstacle in obtaining a tissue sample to diagnose some SETs. A mucosal incision-assisted tissue sampling for gastric SETs is relatively simple and can be performed by endoscopists familiar with endoscopic mucosal resection techniques. As the proper tumor exposure beneath the mucosa is a key element to obtain an adequate tissue sample to diagnose mucosal incision-assisted SET sampling, the degree of technical difficulty should be predicted to overcome possible obstacles while exposing SETs during or after the mucosal incision before attempting this technique. The mucosal incision-assisted endoscopic biopsy is an efficient option for obtaining tissue samples to diagnose gastric SETs.

Conflicts of Interest

The authors have no financial conflicts of interest.

Author Contributions

Writing-original draft: Cheol Woong Choi

Writing-review\&editing: Joo Ha Hwang

ORCID

Cheol Woong Choi: https://orcid.org/0000-0001-8867-3039

\section{REFERENCES}

1. Hwang JH, Rulyak SD, Kimmey MB. American Gastroenterological Association Institute technical review on the management of gastric subepithelial masses. Gastroenterology 2006;130:2217-2228.

2. Lim TW, Choi CW, Kang DH, Kim HW, Park SB, Kim SJ. Endoscopic ultrasound without tissue acquisition has poor accuracy for diagnosing gastric subepithelial tumors. Medicine (Baltimore) 2016;95:e5246.

3. Hwang JH, Saunders MD, Rulyak SJ, Shaw S, Nietsch H, Kimmey MB. A prospective study comparing endoscopy and EUS in the evaluation of GI subepithelial masses. Gastrointest Endosc 2005;62:202-208.

4. Pih GY, Kim DH. Endoscopic ultrasound-guided fine needle aspira- 
tion and biopsy in gastrointestinal subepithelial tumors. Clin Endosc 2019;52:314-320.

5. Nakano Y, Takao T, Morita Y, et al. Reasons for diagnostic failure in forty-five consecutive mucosal cutting biopsy examinations of gastric subepithelial tumors. Clin Endosc 2020;53:575-582.

6. Dhaliwal A, Kolli S, Dhindsa BS, et al. Clinical efficacy and safety of mucosal incision-assisted biopsy for the diagnosis of upper gastrointestinal subepithelial tumors: a systematic review and meta-analysis. Ann Gastroenterol 2020;33:155-161.

7. Choi CW, Kang DH, Kim HW, et al. Direct endoscopic biopsy for subepithelial tumor larger than $20 \mathrm{~mm}$ after removal of overlying mucosa. Scand J Gastroenterol 2017;52:779-783.
8. de la Serna-Higuera C, Pérez-Miranda M, Díez-Redondo P, et al. EUS-guided single-incision needle-knife biopsy: description and results of a new method for tissue sampling of subepithelial GI tumors (with video). Gastrointest Endosc 2011;74:672-676.

9. Minoda Y, Chinen T, Osoegawa T, et al. Superiority of mucosal incision-assisted biopsy over ultrasound-guided fine needle aspiration biopsy in diagnosing small gastric subepithelial lesions: a propensity score matching analysis. BMC Gastroenterol 2020;20:19.

10. Park J, Park JC, Jo JH, et al. Prospective comparative study of endoscopic ultrasonography-guided fine-needle biopsy and unroofing biopsy. Dig Liver Dis 2019;51:831-836. 